\title{
Size dependence of magnetic characteristics measured on separate nickel particles
}

\author{
S. A. Nepijko ${ }^{1}$, R. Wiesendanger ${ }^{2}$ \\ ${ }^{1}$ Institute of Physics, National Academy of Siences of Ukraine, 46, Prospect Nauki,252022 Kiev, Ukraine \\ ${ }^{2}$ Institute of Applied Physics and Microstructure Research Center, University of Hamburg, Jungiusstraße 11, D-20355 Hamburg, Germany
}

\begin{abstract}
Interference electron microscopy was applied to measure the coercive force, the magnetic saturation and the residual magnetization of separated nickel particles. Nickel particles with perfect sphericity and radius from 10 to $100 \mathrm{~nm}$ were produced directly in the interference electron microscope by means of wire explosion caused by the passage of an electric current pulse through it. We find a decrease of the magnetic saturation and an increase of the coercive force with decreasing size of the separate particles. If there are neighbouring particles, with decreasing the distance to them coercive force is characterized by more smooth size dependence and has less absolute value. This observation shows the contribution of the interparticle interaction.
\end{abstract}

Keywords: ferromagnetic particles, size dependence, coercive force, magnetic saturation, residual magnetization

Paper received 25.05.99; revised manuscript received 01.10.99; accepted for publication 12.07.99.

\section{Introduction}

Measurements of magnetic properties of separate small particles is of particular interest. These allow one to distinguish contribution of size-dependent properties from contribution of effects caused by interaction between particles. To do this, an interference electron microscope (IEM) can be applied [1]. Experiments on observation of magnetic structure of Ni particles [2-4] as well as measurement of the temperature dependence of the Curie temperature of Ni particles [5] were performed by means of this technique.

In the present paper, size dependence of a several magnetic characteristics, such as the coercivity, the magnetization of saturation and the remanent magnetization, were measured by IEM. This study was carried out on small Ni particles.

We do not contrast the interference electron microscopy with the Lorentz microscopy [6] and the scanning magneticforce microscopy (MFM) [7] which are also informative when the magnetic properties of separate particles are under investigation. On the contrary, comparison of results obtained by these methods is of interest in the field of overlapping their potentialities.

\section{Experimental}

The study was conducted in a $100 \mathrm{kV}$ IEM with a thermionic cathode. To raise the spatial coherence of the electron beam, it was extended by means of the condensing lenses.
Then it was strongly narrowed by a diaphragm, so that used area of the cathode comprised $100 \mathrm{~nm}$. As a result, the beam intensity was weak, and the exposure time under shooting was long and accounted for 10-20 s. The length of coherence was sufficiently large, it was equal near $0,5 \mathrm{~m}$. An electrostatic analogue of the Fresnel biprism $[8,9]$ was applied in our IEM. For this purpose, a filament $0,7 \mu \mathrm{m}$ in diameter was installed under the lower focal plane of the microscope objective lens normal to its optical axis. Since the filament diameter is less than one of the coherent electron beam, the filament divides this beam into two parts. The part of beam, passing on the one side of the filament, serves as the probe beam. The part of beam on the other side of the filament was used as the reference beam. Trajectories of electrons, passing on the both sides of the filament, are bent towards each other if a positive potential is applied to the filament. The width of the shadow from the filament at the IEM screen decreases as $U_{b}$ increases. In our case the beams overlap at $U_{b} \geq 3 \mathrm{~V}$. The interference fringes were observed in the overlap region. The higher is the voltage applied to the filament, the more is the overlap region. When the amount and spatial density of the fringes grow, distance between them reduces [10]. A settled phase shift $2 \pi$ corresponds to this distance. Thus, as a voltage applied to the filament grows, modulus of bending of the interference fringes, caused by the phase shift $\Delta \Phi$ of the probe beam relative to the reference one, diminishes. Because of this, the filament voltage was also limited from above, and in our case it was equal to $3 \mathrm{~V}<U_{b}<10 \mathrm{~V}$. 


\section{S. A. Nepijko, R. Wiesendanger: Size dependence of magnetic characteristics measured...}

Ni particles were produced direct by in IEM by means of wire explosion caused by the passage of an electric current pulse through it [11]. A source of this kind can be made rather small in size in order to place it in limited free space under the IEM objective lens. Moreover, this source produces particles of near ideal spherical shape that is of great importance because magnetic properties of small particles depend strongly on their shape. Sizes of particles were varied by the pulse duration, the value of current passing through the filament and its diameter. In films prepared in such way, the areas can be found which contain particles well separated from each other. In that case, it's possible to neglect the probable interparticle interaction. At last, employment of the explosion method makes it possible to hope that prepared nickel particles are clean and perfect enough in spite of the fact that they were prepared not in ultrahigh vacuum. The vacuum in the sample area was $10^{-7}$ mbar.

A $10-\mathrm{nm}$ thick carbon film on the copper grid served as a substrate. The substrate electrical conductivity excluded from charging of the particles concerned under the electron beam [12]. The carbon film rolled up in places where there were its ruptures. Particles lying on these rolled regions (Fig.1) were chosen for investigation.

A specimen was somewhat lifted relative to its standard position in the objective lens. This lowered resolution by an order of magnitude, and it amounted to $4-5 \mathrm{~nm}$. However, this enabled the specimen to be placed between two diminutive solenoidal coils, the axis of which intersects normally to the IEM optical axis. Value of the local magnetic field, created by the coils, varied in the range $0 \leq H \leq$ $10^{3}$ Gs (from 0 up to $1,5 \cdot 10^{3} \mathrm{Gs}$ ), but it reach $10^{4} \mathrm{Gs}$ in pulse. The latter exceeded the magnetic field of the objective lens close to the specimen and was used in order to orientate the magnetic moment of the particles under study in the plane normal to the IEM optical axis.

\section{Experimental results and their discussion}

A spherical $\mathrm{Ni}$ particle obtained by the $\mathrm{Ni}$ wire explosion was chosen on the rolled section of the carbon surface. Its magnetic moment was settled in the plane normal to the IEM optical axis by means of a pulse field produced by the solenoids. The following measurements were performed in the constant external uniform magnetic field oriented, as in the case of the pulse field, along the solenoid axis in the direct or opposite direction.

The uniform magnetic field does not result in bending of the interference fringes because the probe and reference coherent electron beams get the same phase in this field. However, in the uniform magnetic field a ferromagnetic particle obtains the magnetic moment that leads to nonuniformity of the magnetic field in its vicinity. Then the coherent electron beams pass through regions with different magnetic fields, and the phase difference arises between them that causes the interference fringes to bend. It is necessary that the magnetic moment of a particle has component normal to the direction of electron motion in the beam [1]. If the magnetic moment of a particle is paral-

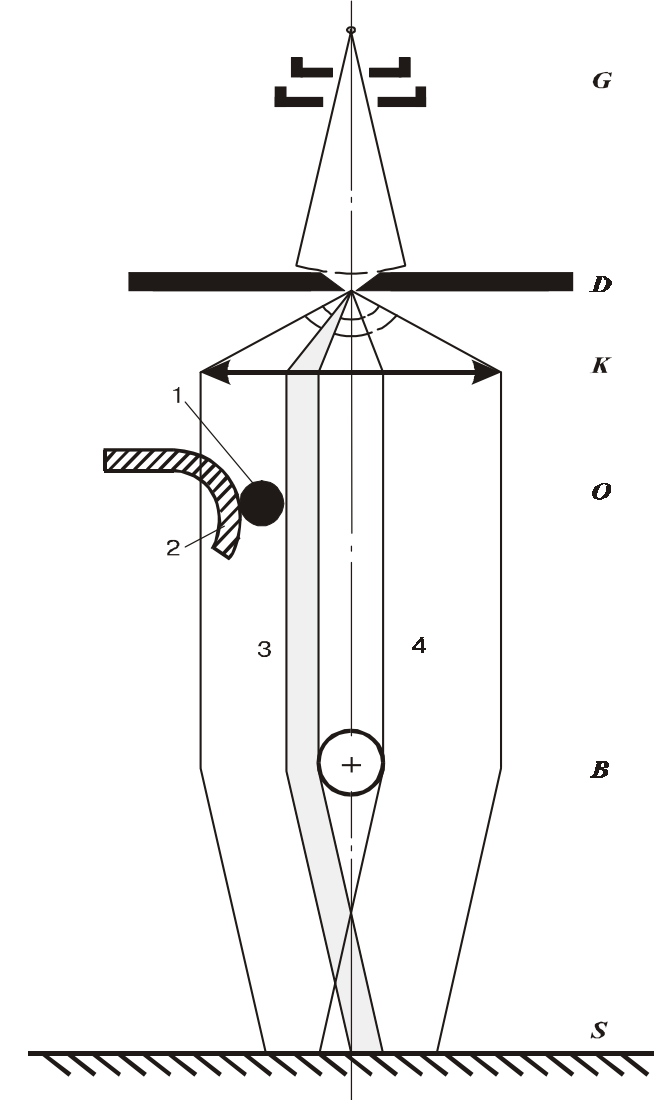

Fig.1. A schematic of the spherical particle under study (1), the carbon substrate with roll (2), the probing (3) and reference (4) coherent electron beams and the IEM elements: illuminating system consisting of $G$ - source of electrons, $D$ - diaphragm, $K$ - condencing lens, $B$ - filament with a positive potential applied to it (an electrostatic analog of the Fresnel biprism), $S$ - screen. Objective, magnifying and projective lenses are not shown.

lel to the electron beam, then the positive phase shift under movement of electron toward this particle is completely compensated by the phase shift of opposite sign under electron moving away from the particle (in this case the picture is entirely symmetrical). A character of bending of the interference fringes is analyzed in ref.[1]. When the external magnetic field enhances, the magnetic moment of the ferromagnetic particle grows as well, and this growth has saturation. Accordingly, the bend of these interference fringes increases with saturation, too. In experiment the external magnetic field enhanced slowly till changes in bending the interference fringes were still observed. We are interested not in the value of external magnetic field itself, but in value and shape of bending the interference fringes out of the particle involved. From these data using Eqs.(15), (4) and (3) from ref.[1] the magnetic moment of the particle in saturation can be calculated as well as the magnetic moment per unit of the volume of particle, i.e. the magnetization of saturation $I_{s}$.

After it the current through the solenoids as well as the external magnetic field went down to zero. The magnetic 


\section{S. A. Nepijko, R. Wiesendanger: Size dependence of magnetic characteristics measured...}

moment, calculated from bending of the interference fringes at disconnected external magnetic field, associates with the remanent magnetization $I_{r}$.

To measure one more characteristic of magnetic reversal, the current was passed through the solenoids in the opposite direction and increased. When the opposing external magnetic field is applied, and the interference fringes bend in the opposite direction, the value of this field is the coercivity $H_{c}$.

Measurements of three characteristics of the magnetization curve (hysteresis loops) were carried out on $\mathrm{Ni}$ particles of different sizes, results are presented in Fig.2a-c. The coercivity $H_{c}$ rises when the radius $R$ of $\mathrm{Ni}$ particles decreases, i.e. the coercivity is size-dependent. The coercivity tends to diminish with increasing distance between particles. The experimental points in Fig.1 are in the hatched region for this case. Measurements of value of the coercivity do not require any recalculation of form of the experimentally observed interference fringes, i.e. they are characterized by high precision. It falls as the concentration of particles grows. Really, if there are adjacent particles, the effective magnetic field, switching the magnetic moment of particles, is already poorly determined because it is a superposition of the external field and the magnetic fields of adjacent particles.

Measured dependence $H_{c}(R)$ is understandable qualitatively. Indeed, decrease of sizes of ferromagnetic particles is accompanied by reconstruction of their magnetic structure. In this case, when transition from multi-domain to mono-domain state takes place, possibility of nucleation of magnetic reversal centers decreases that leads to rise of the coercivity.

The value of demagnetizing factor changes with increasing concentration of the ferromagnetic particles because of their interaction, which is mainly magnetostatic. As a result, the critical size of the mono-domain state $R_{0}$ [13] grows. Therefore, a maximum of the dependence $H_{c}(R)$ shifts towards greater sizes. This results in the fact that at a settled particle size $\left(R>R_{0}\right)$ the coercivity should rise as the bulk concentration of particles increases. It is also clear that the magnetic reversal of particles in ensemble has some peculiar features. Fig.3a-d illustrates schematically possible types of the magnetic reversal in a separate particle and their

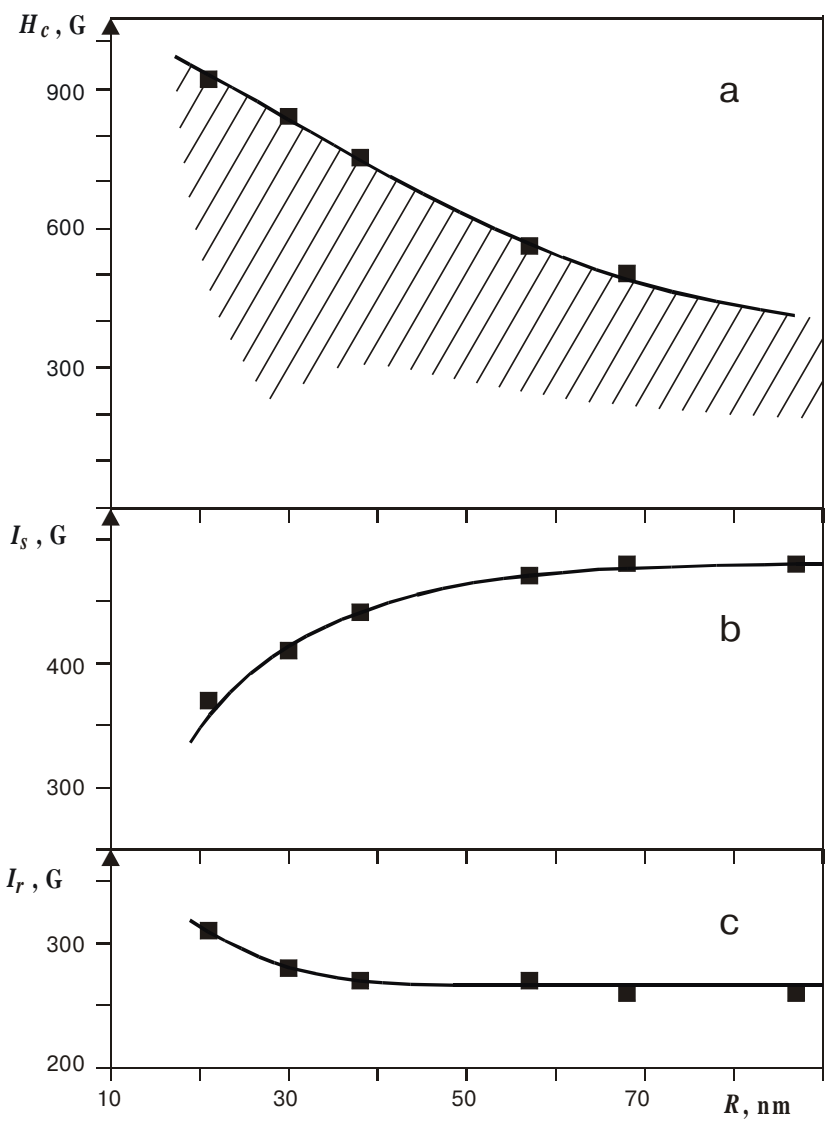

Fig.2. Size dependencies of (a) the coercivity $H_{c}(R)$; (b) the magnetization of saturation $I_{S}(R)$ and (c) the remanent magnetization $I_{r}(R)$. They were measured on separate Ni particles of radius $R$. In the hatched region there are experimental points for the case when there are neighboring particles close to the particle under study.

ensembles that is discussed in literature. When distance between particles reduces, type of the magnetic reverse changes from twisting (Fig.3a) to parallel rotation of the elementary magnetic moments in particles (Fig.3b) [14,15]. In this case the simplest consideration of character of magnetic reversal of mono-domain neighboring particles supposes that all their magnetic moments turn simultaneously and coherently (Fig.3c). It proved to be reasonable that the

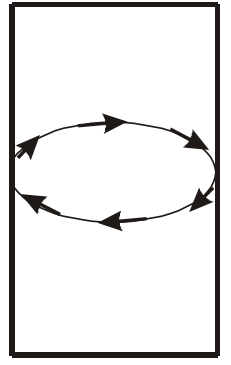

a

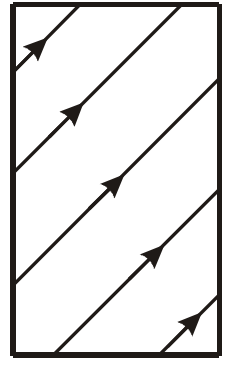

$b$

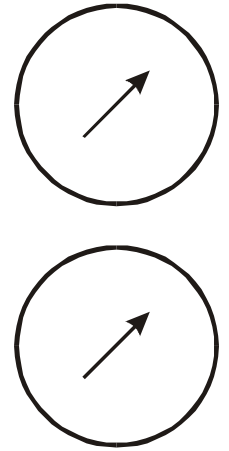

C

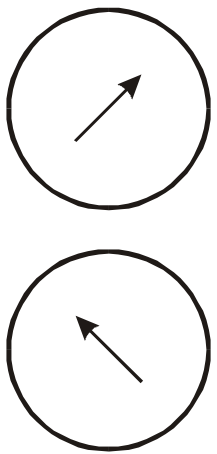

d

Fig.3. Possible types of the magnetic reversal of mono-domain particles isolated by twisting (a) and parallel rotation (b) as well as in the case of their ensemble. The magnetic reversal can be both coherent (c) and incoherent (d). 


\section{S. A. Nepijko, R. Wiesendanger: Size dependence of magnetic characteristics measured...}

coercivity of a chain of spherical particles reversing the magnetism according to this scheme was much more than in the case of a separate particle [16]. However, under the action of external field the vectors of magnetic moments can also turn to opposite direction in this way, and that is shown in Fig. 3d. This process is preferable in terms of energetics [16]. The real situation is certainly more complicated because of multidomain state of particles, size dispersion of particles and dispersion of distances between them. Our experimental measurements show a rise of the coercivity with decreasing distance between particles (Fig.2a, hatched region). This strongly supports the model of coherent magnetic reversal.

Measurement of the magnetization of saturation $I_{S}$ is characterized by the maximum sensitivity when the absolute value of the bend of above interference fringes is maximum. As illustrated in Fig.1b, the magnetization of saturation $I_{s}$ tends to diminish with decreasing radius of particles $R$. In refs.[17-21] the suggestion has been made that similar behavior of the magnetization of saturation of the small ferromagnetic particles, i.e. of the magnetic moment of volume unit, can be caused by oxidation of their surface. In our case, Ni particles were produced directly in the IEM column, i.e. they were not transferred via atmosphere. However, since vacuum in the region close to specimen comprised $10^{-7} \mathrm{mbar}$, the particles could partly oxidize. One should take into consideration that the size dependence of the magnetization of saturation $I_{s}=I_{s}^{(\infty)}(1-a / R)^{3}$ describes results of the experimental measurements only under a rather strange condition of an increasing depth of the oxidized layer with increasing sizes of particles. Hence it follows that oxidation is not the only reason of lowering the magnetization of saturation with decreasing sizes of particles. The same objections are also true when an idea about chemisorption of, for example, hydrogen is proposed [22] or existence is assumed of «dead» atom layers on the particle surface which do not take part in ferromagnetism [23,24]. Relying on these reasons, a conclusion can be drawn that dependence of the magnetization of saturation on the particles' sizes is not a result of their oxidation. It seems reasonable to say that as the particle size diminishes, more and more significant part of spins adjoining the surface has a stable direction which does not coincide with the direction along which spins are lined up in the bulk of the particle. As a result, two spin subsystems arise in the small ferromagnetic systems. One of them is collinear in the bulk, and in near-surface layer the second subsystem has a component of magnetization normal to the spin orientation of the first one (Fig.4).

Results of measuring the remanent magnetization $I_{r}$ are presented in Fig.1c. Because of small accuracy and sensitivity of these measurements, it is possible to say only about a tendency of $I_{r}$ growth with decreasing radius of particles $R$. This tendency appears to be correct. Indeed, as the particles' sizes reduce, $I_{s}$ diminishes as well, i.e., the height of hysteresis loop is lowered. However, since its area remains constant, it follows that $I_{r}$ rises.

In measurements of the magnetic characteristics of separate particles by means of the interference electron micros-

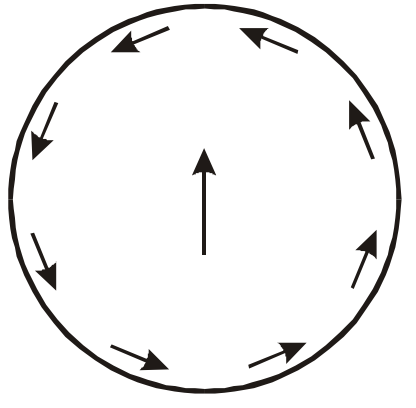

Fig.4. A model representation of the domain structure of a small particle having two spin subsystems - in bulk and in near-surface layer.

copy the value of the bend of the interference fringes does not exceed several percent from the distance between adjacent fringes. In order to register so small bending, the following steps were taken:

- spherical particles on the substrate roll were chosen for measurements. If the probing beam and, especially, if both the probing and reference coherent beams intersect the substrate, then, because of its heterogeneity in thickness and density, fluctuations of phase comprise already several percent from $2 \pi$ (the phase shift between two adjacent interference fringes), i.e., they are comparable with the measured signal;

- bending the interference fringes was analyzed out of a particle, but not on its image. Out of Ni particle bending the interference fringes is caused by its magnetic moment, whereas on the particle image bending is stimulated by the magnetic moment as well as by the inner potential. The second effect is considerably stronger [1]. In this case measurements are not limited by arbitrary large sizes of particles;

- effect of bending the interference fringes caused by charging of a particle under the electron beam [12] was excluded by using a substrate with good conductivity;

- the value of interference fringes' bending was determined from conditions that their shape is described by the expression (15) from ref.[1] and sum of least squares. It should be noted here that it does not matter how the orthogonal axes $\mathrm{O} x$ and $\mathrm{O} y$ are lined in the interferogram plane. Mutual orientation of the direction of external magnetic field and of the biprism filament is of no importance, too.

\section{Conclusion}

Measurements of the size dependence of the coercivity $H_{c}(R)$, the magnetization of saturation $I_{s}(R)$ and the remanent magnetization $I_{r}(R)$ were performed on separate Ni particles. This allows judging about the value and character of deformation of the hysteresis loop as sizes of Ni particles decrease. $H_{c}$ and $I_{r}$ increase and $I_{s}$ decreases with diminishing sizes of Ni particles. Influence of interaction of particles with each other on the value of their magnetic characteristics has been shown. In particular, $H_{c}$ goes down as concentration of Ni particles rises. 


\section{S. A. Nepijko, R. Wiesendanger: Size dependence of magnetic characteristics measured...}

\section{References}

1. S.A.Nepijko, R.Wiesendanger: Appl. Phys. A 65, 361 (1997)

2. A.Tonomura, T.Matsuda, J.Endo: Phys. Rev. Lett. 44, 1430 (1980)

3. T.Matsuda, A.Tonomura, R.Suzuki, J.Endo, N.Osakabe, H.Umezaki, H.Tanabe, Y.Siguta, H.Fujiwara: J. Appl. Phys. 53, 5444 (1982)

4. J.N.Chapman, R.P.Ferrier, U.J.Heyderman, S.McVitie, W.A.P.Nicholson, B.Bormans: Inst. Phys. Conf. Ser. No.138: Section 1 (Proc. Electron Microscopy and Analysis Group Conf. EMAG93, Liverpool, 1993) P.1-8

5. S.A.Nepijko, R.Wiesendanger: Europhys.Lett. 31, 567 (1995)

6. T.Arii, S.Yatsuya, N.Wada, K.Mihama: Jap. J. Appl. Phys. 17, 259 (1978)

7. A.A.Bukharaev, D.A.Ovchinnokov, N.I.Nurgazizov, E.F.Kukovitskii, M.Kleiber, R.Wiesendanger: Phys. Solid State, 40, 1163 (1998)

8. G.Mullenstedt, H.Dıker: Z. Physik 145, 377 (1956)

9. V.Dragos, A.Delong: Cesk. Cas. Fys. 13, 278 (1963); Slaboproudy obsor 25, 523 (1964)

10. I.G.Stoyanova, I.F.Anaskin: Physical principles of transmission electron microscopy, Nauka, Moscow 1972. In Russian

11. V.N.Byckov, Yu.A.Kulyupin, A.A.Lisenko, S.A.Nepijko: Fiz. Met. \& Metalloved. (USSR) 52, 1176 (1981). In Russian. English translation in: Phys. Met. \& Metallogr. (GB)

12. V.N.Byckov, Yu.A.Kulyupin, S.A.Nepijko, E.A.Pashitskii: Radiotekhnika \& Elektronika, 26, 1055 (1981). In Russian
13. S.A.Nepijko: Physical properties of small metal particles, Naukova Dumka, Kiev 1985. In Russian

14. A.Aharoni: phys. status solidi 16, 3 (1966)

15. A.Aharoni: J. Appl. Phys. 35, 347 (1964)

16. I.S.Jacobs, C.P.Bean: Phys. Rev. 100, 1060 (1955)

17. C.R.Abeledo, P.W.Selwood: J.Appl. Phys. 32, suppl. 229S (1961)

18. A.Tasaki, S.Tomiyama, S.Iida, R.Uyeda: Jap. J. Appl. Phys. 4, 707 (1965)

19. Yu.I.Petrov, Yu.I.Fedorov: Zh. Tekh. Fiz. (USSR) 37, 726 (1967). In Russian. English translation in: Soviet Phys. Tech. Phys. (USA) 12, (1967)

20. A.E.Ermakov, O.A.Ivanov, Ya.S.Shur, R.M.Grechishkin, G.V.Ivanova: Fiz. Met. \& Metalloved. (USSR) 33, 558 (1972). In Russian. English translation in: Phys. Met. \& Metallogr. (GB)

21. I.N.Shabanov, A.E.Ermakov, V.A.Trapeznikov, Ya.S.Shur: Fiz. Met. \& Metalloved. (USSR) 38, 314 (1974). In Russian. English translation in: Phys. Met. \& Metallogr. (GB)

22. P.W.Selwood: Adsorption and collective paramagnetism, Acad. Press, New York 1962

23. L.Lieberman, J.Clinton, D.M.Edwards, J.Mathon: Phys. Rev. Lett. 25, $232(1970)$

24. L.N.Liebermann, D.P.Fredkin, H.B.Chore: Phys. Rev. Lett. 22, 539 (1969) 